N. Tu Cuong

Nagoya Math. J.

Vol. 120 (1990), 77-88

\title{
ON THE LENGTH OF THE POWERS OF SYSTEMS OF PARAMETERS IN LOCAL RING
}

\author{
NGUYEN TU CUONG
}

\section{$\S 1$. Introduction}

Throughout this note, $A$ denotes a commutative local Noetherian ring with maximal ideal $\mathfrak{m}$ and $M$ a finitely generated $A$-module with $\operatorname{dim}(M)$ $=d$. Let $x_{1}, \cdots, x_{d}$ be a system of parameters (s.o.p. for short) for $M$ and $I$ the ideal of $A$ generated by $x_{1}, \cdots, x_{d}$. We consider the length $l\left(M /\left(x_{1}^{n_{1}}, \cdots, x_{d}^{n_{d}}\right) M\right)$ over $A$ as a function in the positive integers $n_{1}, \cdots, n_{d}$. J-L. Garcia Roig and D. Kirby [5] have shown that this function is generally not a polynomial for $n_{1}, \cdots, n_{d} \gg 0$ (sufficiently large) but, if $M$ is a generalized Cohen-Macaulay module, then

$$
l\left(M /\left(x_{1}^{n_{1}}, \cdots, x_{d}^{n_{d}}\right) M\right)=n_{1} \cdots n_{d} e(I ; M)+\sum_{i=0}^{d-1}\left(\begin{array}{c}
d-1 \\
i
\end{array}\right) l\left(H_{\mathrm{m}}^{i}(M)\right)
$$

for $n_{1}, \cdots, n_{d} \gg 0$, where $e(I ; M)$ denotes the multiplicity of $M$ relative to $I$ and $H_{\mathrm{m}}^{i}(M)$ is the $i$-th local cohomology module of $M$ with respect to $\mathrm{m}$. Therefore, it is natural to ask under which conditions $l\left(M /\left(x_{1}^{n_{1}}, \cdots, x_{d}^{n_{d}}\right) M\right)$ is a polynomial for $n_{1}, \cdots, n_{d} \gg 0$ ? (see [9], Question 1.1).

The purpose of this note is to give an answer to this question. Before stating the main result we need the following definition. Let $x_{1}, \cdots, x_{d}$ be a s.o.p. for $M$. We say that $x_{1}, \cdots, x_{d}$ is a $p$-system of parameters ( $p$-s.o.p. for short) for $M$ if there exists a positive integer $n_{0}$ such that

$$
\left(x_{1}^{n_{1}}, \cdots, x_{i-1}^{n_{i-1}}\right) M: x_{i}^{n_{i}}=\left(x_{1}^{n_{1}}, \cdots, x_{i-1}^{n_{i-1}}\right) M: x_{i}^{n_{0}}
$$

for all $n_{1}, \cdots, n_{d} \geq n_{0}, i=1, \cdots, d\left(x_{0}=0\right)$.

We say that $x_{1}, \cdots, x_{d}$ is an unconditioned $p$-s.o.p. if for every permutation of the sequence $x_{1}, \cdots, x_{d}$, the above condition holds with respect to the same integer $n_{0}$.

THEOREM 1. The function $l\left(M /\left(x_{1}^{n_{1}}, \cdots, x_{d}^{n_{a}}\right) M\right)$ is a polynomial for Received July 21, 1989. 
$n_{1}, \cdots, n_{d} \gg 0$ if and only if $x_{1}, \cdots, x_{d}$ is an unconditioned p-s.o.p. for $M$.

We will prove this theorem in Section 2. In Section 3, we will relate p-s.o.p.'s to some special s.o.p.'s in the theory of local ring such as filter regular s.o.p.'s [3] and standard s.o.p.'s [11] and show that a generalized Cohen-Macaulay module can be characterized by $p$-s.o.p.'s. At the end of this note, in Section 4, we consider the case $\operatorname{dim}(M)=2$ more closely. In this case, we will see that a $p$-s.o.p. can be characterized by the finitely generated condition of certain relative Rees ring by using a recent result of $P$. Schenzel (see [6]).

\section{§ 2. Proof of Theorem 1}

For convenience, we will use following notations.

Let $n_{1}, \cdots, n_{d}$ be positive integers. Then we put $n(i)=\left(n_{1}, \cdots, n_{i}\right)$ and $I_{n(i)}=\left(x_{1}^{n_{1}}, \cdots, x_{i}^{n_{i}}\right) A, I=I_{d}=\left(x_{1}, \cdots, x_{d}\right) A$.

If $\alpha$ is an element of the group of permutations $S_{d}, \alpha=(\alpha(1), \cdots, \alpha(d))$ we set $I_{n(\alpha(i))}=\left(x_{\alpha(1)}^{n_{\alpha(1)}}, \cdots, x_{\alpha(i)}^{n_{\alpha(i)}}\right) A$.

We first note that if a s.o.p. $x_{1}, \cdots, x_{d}$ for $M$ is a regular $M$-sequence then it is obviously an unconditioned p-s.o.p.. Furthermore, if $x_{1}^{n_{1}}, \cdots, x_{d}^{n_{d}}$ is a $d$-sequence which has been introduced by Huneke [13] for all $n_{1}, \cdots$, $n_{d} \gg 0$ then $x_{1}, \cdots, x_{d}$ is a $p$-s.o.p. Therefore, by [3], every s.o.p. for a generalized Cohen-Macaulay module is an unconditioned $p$-s.o.p. The property of $p$-s.o.p.'s has been examined more closely by the author in [12]. Here, we only give some characterizations of unconditioned p-s.o.p.'s which we need for the proof of Theorem 1.

Lemma 2. The following conditions are equivalent:

(i ) $x_{1}, \cdots, x_{d}$ is an unconditioned p-s.o.p.

(ii) There exists a positive integer $n_{0}$ such that for all $n_{1}, \cdots, n_{d} \geq n_{0}$ and any permutation $\alpha$ of $S_{d}$, we have the equality

$$
\left(I_{n(\alpha(i-1))} M: x_{\alpha(i)}^{\left.n_{\alpha(i)}\right)} \cap I_{n(\alpha(i))} M=I_{n(\alpha(i-1))} M,\right.
$$

for each $i=1, \cdots, d$.

(iii) There exists a positive integer $n_{0}$ such that for all $n_{1}, \cdots, n_{d} \gg n_{0}$ and any permutation $\alpha$ of $S_{d}$, we have the equality

$$
\left(I_{n(\alpha(d-1))} M: x_{\alpha(d)}^{n_{\alpha}(d)}\right) \cap I_{n(d)} M=I_{n(\alpha(d-1))} M .
$$

(iv) There exists a positive integers $n_{0}$ such that for all $n_{1}, \cdots, n_{d} \gg n_{0}$ 
and any permutation $\alpha$ of $S_{a}$, we have the equality

$$
I_{n(\alpha(d-1))} M: x_{\alpha(d)}^{n_{\alpha}(d)}=I_{n(\alpha(d-1))} M: x_{\alpha(d)}^{n_{0}} .
$$

Proof. (i) $\Rightarrow$ (ii). By renaming the permuted sequence it suffices to show that

$$
\left(I_{n(i-1)} M: x_{i}^{n_{2}}\right) \cap I_{n(i)} M \subseteq I_{n(i-1)} M .
$$

Let $a \in\left(I_{n(i-1)} M: x_{i}^{n_{i}}\right) \cap I_{n(i)} M$. Write $a=\sum_{j=1}^{i} y_{j} x_{j}^{n_{j}}$ for some $y_{j} \in M$. Since $a x_{i}^{n_{i}} \in I_{n(i-1)} M$ therefore $y_{i} x_{i}^{2 n_{i}} \in I_{n(i-1)} M$. Hence, for $n_{i} \geq n_{0}, y_{i} \in I_{n(i-1)} M: x_{i}^{2 n_{i}}$ $=I_{n(i-1)} M: x_{i}^{n_{i}}$. It follows that $y_{i} x_{i}^{n_{i}} \in I_{n(i-1)} M$ and $a \in I_{n(i-1)} M$.

(ii) $\Rightarrow$ (iii) is trivial.

(iii) $\Rightarrow$ (iv). For $n_{1}, \cdots, n_{d} \geq n_{0}$ we have

$$
\left(I_{n(d-1)} M: x_{d}^{n_{0}}\right) \cap\left(I_{n(d-1)}+x_{d}^{n_{0}}\right) M=I_{n(d-1)} M .
$$

Dividing both sides of this relation by $x_{d}^{n_{0}}$, we get

$$
I_{n(d-1)} M: x_{d}^{2 n_{0}}=I_{n(d-1)} M: x_{d}^{n_{0}} .
$$

From this we can deduce that

$$
I_{n(a-1)} M: x_{d}^{n_{0}}=I_{n(d-1)} M: x_{d}^{k n_{0}}
$$

for all $n_{1}, \cdots, n_{d} \geq n_{0}$ and $k \geq 1$. Since our proof is independent of the order of the sequence $x_{1}, \cdots, x_{d}$ we obtain (iv).

(iv) $\Rightarrow$ (i). By Krull's Intersection Theorem and (iv) we get

$$
\begin{aligned}
I_{n(i-1)} M: x_{i}^{n_{i}} & \subseteq \bigcap_{k=n_{0}}^{\infty}\left(\left(I_{n(i-1)}+x_{i+1}^{k}+\cdots+x_{d}^{k}\right) M: x_{i}^{n_{i}}\right) \\
& =\bigcap_{k=n_{0}}^{\infty}\left(\left(I_{n(i-1)}+x_{i+1}^{k}+\cdots+x_{d}^{k}\right) M: x_{i}^{n_{0}}\right) \\
& =I_{n(i-1)} M: x_{i}^{n_{0}} \subseteq I_{n(i-1)} M: x_{i}^{n_{i}}
\end{aligned}
$$

for each $i=1, \cdots, d$ and all $n_{1}, \cdots, n_{d} \geq n_{0}$. Since the proof is independent of the order of the sequence $x_{1}, \cdots, x_{d}$ we conclude Lemma 2 .

LEMMA 3. If $l\left(M / I_{n(d)} M\right)$ is a polynomial for $n_{1}, \cdots, n_{d} \gg 0$ then it is linear in each $n_{i}$.

Proof. Straightforward.

The following formula for the multiplicity, found by Auslander and Buchsbaum (see $[1, \S 4]$ ), is the starting point for the proof of Theorem 1 .

LEMMA 4. For every s.o.p. $x_{1}, \cdots, x_{d}$ for $M$ we have 


$$
l\left(M /\left(x_{1}, \cdots, x_{d}\right) M\right)=l\left(I_{d-1} M: x_{d} / I_{d-1} M\right)+\sum_{i=0}^{d-1} e\left(I_{d} / I_{i} ; I_{i-1} M: x_{i} / I_{i-1} M\right),
$$

where $e\left(I_{d} / I_{i} ; I_{i-1} M: x_{i} / I_{i-1} M\right)=e\left(I_{d} ; M\right)$ for $i=0$.

Proof of Theorem 1. $(\Rightarrow)$. Using Lemma 4 we have

$$
\begin{aligned}
& l\left(M / I_{n(d-1)} M+x_{d}^{k n_{d}} M\right)-l\left(M / I_{n(d)} M\right) \\
&= l\left(I_{n(d-1)} M: x_{d}^{k n_{d}} / I_{n(d-1)} M\right)-l\left(I_{n(d-1)} M: x_{d}^{n_{d}} / I_{n(d-1)} M\right) \\
&+\sum_{i=0}^{d-1} e\left(I_{n(d-1)}+x_{d}^{(k-1) n_{d}} A / I_{n(i)} ; I_{n(i-1)} M: x_{i}^{n_{d}} / I_{n(i-1)} M\right) \\
&= l\left(I_{n(d-1)} M: x_{d}^{k n_{d}} / I_{n(d-1)} M\right)-l\left(I_{n(d-1)} M: x_{d}^{n_{d}} / I_{n(d-1)} M\right) \\
&+l\left(M / I_{n(d-1)} M+x_{d}^{(k-1) n_{d}} M\right)-l\left(I_{n(d-1)} M: x_{d}^{(k-1) n_{d}} / I_{n(d-1)} M\right)
\end{aligned}
$$

for all positive integers $k$. For arbitrary $n(d-1)=\left(n_{1}, \cdots, n_{d-1}\right)$, we can find $k$ (in general depending on $n_{1}, \cdots, n_{d-1}$ ) such that

$$
I_{n(d-1)} M: x_{d}^{k n_{d}}=I_{n(d-1)} M: x_{d}^{(k-1) n_{d}} .
$$

Thus,

$$
\begin{aligned}
& l\left(I_{n(d-1)} M: x_{d}^{n_{d}} / I_{n(d-1)} M\right) \\
& \quad=l\left(M / I_{n(d)} M\right)+l\left(M / I_{n(d-1)} M+x_{d}^{(k-1) n_{d}} M\right)-l\left(M / I_{n(d-1)} M+x_{d}^{k n_{d}} M\right) .
\end{aligned}
$$

By Lemma 3 each summand of the above right term is a polynomial, linear in each $n_{i}$, for all $n_{1}, \cdots, n_{d} \gg 0$. Therefore $l\left(I_{n(d-1)} M: x_{d}^{n_{d}} / I_{n(d-1)} M\right)$ is a polynomial. For fixed $n^{0}(d-1)=\left(n_{1}^{0}, \cdots, n_{d-1}^{0}\right)$, there exists a positive integer $t$ such that

$$
I_{n 0(d-1)} M: x_{d}^{n d}=I_{n 0(d-1)} M: x_{d}^{t}
$$

for $n_{d} \geq t$. This implies that the polynomial $l\left(I_{n(d-1)}\left(M: x_{d}^{n_{d}} / I_{n(d-1)} M\right)\right.$ is independent of $n_{d}$. Hence, there exists a positive integer $n_{0}$ such that $I_{n(d-1)} M: x_{d}^{n_{d}}=I_{n(d-1)} M: x_{d}^{n_{0}}$ for $n_{1}, \cdots, n_{d} \gg n_{0}$. As our proof is independent of the order of the sequence $x_{1}, \cdots, x_{d}$, it follows by Lemma 2 (iv) that $x_{1}, \cdots, x_{d}$ is an unconditioned p-s.o.p. $(\Leftarrow)$. For convenience, we set

$$
l\left(I_{n(d-1)} M: x_{d}^{n_{d}} / I_{n(d-1)} M\right)=e\left(I_{n(d)} / I_{n(d)} ; I_{n(d-1)} M: x_{d}^{n_{d}} / I_{n(d-1)} M\right) .
$$

Then, by Lemma 4, it suffices to show that for each $i=0,1, \cdots, d$, $e\left(I_{n(d)} / I_{n(i)} ; I_{n(i-1)} M: x_{i}^{n_{i}} / I_{n(i-1)} M\right)$ is a polynomial for $n_{1}, \cdots, n_{d} \gg 0$. We will argue by induction on $d$ and $i$.

If $d=1$ or $i=0$ and $d$ arbitrary, the statement is trivial.

It $d>1$ and $i \geq 1$, we suppose that the result is true for $d-1$ or 
$i-1$, and it suffices to show that

$$
e\left(I_{n(d)} / I_{n(i)} ; I_{n(i-1)} M: x_{i}^{n_{i}} / I_{n(i-1)} M\right)
$$

is a polynomial. Consider a permutation $\alpha=(\alpha(1), \cdots, \alpha(d))$ of $S_{d}$ such that $\alpha(i-1)=i, \alpha(i)=i-1$ and $\alpha(j)=j$ for all $j \neq i-1, i$. Then by Lemma 3 and the assumption, there exists a positive integer $n_{0}$ such that

$$
\begin{aligned}
0= & l\left(M / I_{n(d)} M\right)-l\left(M / I_{n(\alpha(d))} M\right) \\
= & \left.e\left(I_{n(d)} / I_{n(i-1)}\right): I_{n(i-2)} M: x_{i-1}^{n_{0}} / I_{n(i-2)} M\right) \\
& +e\left(I_{n(d)} / I_{n(i)} ; I_{n(i-1)} M: x_{i}^{n_{0}} / I_{n(i-1)} M\right) \\
& -e\left(I_{n(d)} / I_{n(\alpha(i-1))} ; I_{n(i-2)} M: x_{i}^{n_{0}} / I_{n(i-2)} M\right) \\
& -e\left(I_{n(d)} / I_{n(\alpha(i))} ; I_{n(\alpha(i-1))} M: x_{i-1}^{n_{0}} / I_{n(\alpha(i-1))} M\right)
\end{aligned}
$$

for all $n_{1}, \cdots, n_{d} \gg n_{0}$. It follows that

$$
\begin{aligned}
e\left(I_{n(d)} / I_{n(i-1)} ; I_{n(i-2)} M: x_{i-1}^{n_{0}} / I_{n(i-2)} M\right) & \\
& -e\left(I_{n(d)} / I_{n(\alpha(i))} ; I_{n(\alpha(i-1))} M: x_{i-1}^{n_{0}} / I_{n(\alpha(i-1))} M\right) \\
= & e\left(I_{n(d)} / I_{n(\alpha(i-1))} ; I_{n(i-2)} M: x_{i}^{n_{0}} / I_{n(i-2)} M\right) \\
& -e\left(I_{n(d)} / I_{n(i)} ; I_{n(i-1)} M: x_{i}^{n_{0}} / I_{n(i-1)} M\right) .
\end{aligned}
$$

Denote the function on the rihgt of the above formula by $F$. Since the above left term is a function independent of $n_{t-1}$, so is $F$. For $n_{1}, \cdots, n_{d}$ $\geq n_{0}$. we have

$$
\begin{aligned}
F= & e\left(x_{i-1}^{n_{0}}, x_{i+1}^{n_{i}+1}, \cdots, x_{d}^{n_{d}} ; I_{n(i-2)} M: x_{i}^{n_{0}} / I_{n(i-2)} M\right) \\
& -e\left(I_{n(d)} / I_{n(i)} ;\left(I_{n(i-2)}+x_{i-1}^{n_{0}}\right) M: x_{i}^{n_{0}} / I_{n(i-2)} M+x_{i-1}^{n_{0}} M\right) .
\end{aligned}
$$

Set $\bar{M}=M / x_{i-1}^{n_{0}} M . \quad$ As $\operatorname{dim}(\bar{M})=d-1$, by induction on $d$, it follows that

$$
\begin{aligned}
e\left(I_{n(d)} / I_{n(i)} ;\left(I_{n(i-2)} M\right.\right. & \left.+x_{i-1}^{n_{0}} M\right): x_{i}^{n_{0}} /\left(I_{n(i-2)}+x_{i-1}^{n_{0}}\right) M \\
& =e\left(I_{n(d)} / I_{n(i)} ; I_{n(i-2)} \bar{M}: x_{i}^{n_{0}} / I_{n(i-2)} \bar{M}\right)
\end{aligned}
$$

is a polynomial for $n_{1}, \cdots, n_{d} \geq n_{0}$. On the other hand, by induction on $i, e\left(x_{i-1}^{n_{0}}, x_{i+1}^{n_{i}+1}, \cdots, x_{d}^{n_{d}} ; I_{n(i-2)} M: x_{\imath}^{n_{0}} / I_{n(i-2)} M\right)$ is a polynomial. Thus, $F$ is a polynomial because $F$ is the difference of two polynomials. Consequently, by induction on $i$,

$$
\begin{aligned}
e\left(I_{n(d)} / I_{n(i)} ;\right. & \left.I_{n(i-1)} M: x_{i}^{n_{0}} / I_{n(i-1)} M\right) \\
& =e\left(I_{n(d)} / I_{n(\alpha(i-1)} ; I_{n(i-2)} M: x_{i}^{n_{0}} / I_{n(i-2)} M\right)+F
\end{aligned}
$$

is a polynomial for all $n_{1}, \cdots, n_{a} \gg n_{0}$. The proof of Theorem 1 is now complete. 
Remark. For the case $n_{1}=\cdots=n_{d}=n$ as treated in [5] Theorem 1 leads to the following questions:

1. Let $x_{1}, \cdots, x_{d}$ be a s.o.p. for $M$. Then $l\left(M /\left(x_{1}^{n}, \cdots, x_{d}^{n}\right) M\right)$ is a polynomial for $n \gg 0$ if and only if $l\left(M /\left(x_{1}^{n_{1}}, \cdots, x_{d}^{n_{d}}\right) M\right)$ is a polynomial for $n_{1}, \cdots, n_{d} \gg 0$ ?

2. Is Theorem 1 still true for this case? That is, $l\left(M /\left(x_{1}^{n_{1}}, \cdots, x_{d}^{n_{d}}\right) M\right)$ is a polynomial for $n_{i} \gg 0$ if and only if there exists a positive integer $n_{0}$ such that, for all $n \geq n_{0}$ and any permutation $\alpha$ of $S_{d}$.

$$
\left(x_{\alpha(1)}^{n}, \cdots, x_{\alpha(i-1)}^{n}\right) M: x_{\alpha(i)}^{n}=\left(x_{\alpha(1)}^{n}, \cdots, x_{\alpha(i-1)}^{n}\right) M: x_{\alpha(i)}^{n_{0}},
$$

for each $i=1, \cdots, d$ ?

Unfortunately, these questions do not always have an affirmative answer as the following example shows: For $d \geq 2$, let $B_{d}=k \llbracket Y_{1}, \cdots, Y_{d+1} \rrbracket$ $/\left(Y_{1} Y_{d+1}, \cdots, Y_{d} Y_{d+1}\right)$, where $k$ is a field and $Y_{1}, \cdots, Y_{d+1}$ are indeterminates. We denote by $x_{i}$ the natural image of $Y_{i}+Y_{d+1}$ in $B_{d}, i=1, \cdots, d$, then $x_{1}, \cdots, x_{d}$ form an s.o.p. for $B_{d}$. It can be verified that

$$
l\left(B_{d} /\left(x_{1}^{n_{1}}, \cdots, x_{d}^{n_{d}}\right) B_{d}\right)=n_{1} \cdots n_{d}+\min \left\{n_{1}, \cdots, n_{d}\right\},
$$

for all $n_{1}, \cdots, n_{d} \geq 1$ and

$$
\left(x_{1}^{n}, \cdots, x_{i-1}^{n}\right) B_{d}: x_{i}^{k}=\left(y_{1}^{n}, \cdots, y_{i-1}^{n}, y_{d+1}\right) B_{d}, \quad i=1, \cdots, d,
$$

for all $k \geq 1$, where $y_{i}$ is the natural image of $Y_{i}$ in $B_{d}$. Therefore $x_{1}, \cdots, x_{d}$ satisfy the condition $(*)$ but $l\left(B_{d} /\left(x_{1}^{n_{1}}, \cdots, x_{d}^{n_{d}}\right) B_{d}\right.$ is not a polynomial.

\section{§3. Generalized Cohen-Macaulay modules}

In this section we will see that p-s.o.p.'s are closely related to some specified s.o.p.'s like filter regular s.o.p.'s [3] or standard s.o.p.'s [11] and that one can use the notation of p-s.o.p.'s to characterize the generalized Cohen-Macaulay module which has been first introduced in [3].

Recall that an s.o.p. $x_{1}, \cdots, x_{d}$ for $M$ is called a filter regular s.o.p. if $x_{i} \notin P$ for all $P \in \operatorname{Ass}\left(M /\left(x_{1}, \cdots, x_{i-1}\right) M-\{\mathfrak{m}\}, i=1, \cdots, d\right.$. It is called an unconditioned filter regular s.o.p. if for any order of the sequence $x_{1}, \cdots, x_{d}$ it is always a filter regular sequence. This notion was introduced in [3] and has led to some interesting results. For instance, $M_{P}$ is a Cohen-Macaulay module and $\operatorname{dim}\left(M_{P}\right)+\operatorname{dim}(A / P)=\operatorname{dim}(M)$ for all $P \in \operatorname{Supp}(M)-\{\mathfrak{m}\}$ if and only if every s.o.p. for $M$ is a filter regular 
s.o.p. [3, Satz 2.5]. In this case, $M$ is called an $f$-module. $M$ is called a generalized Cohen-Macaulay module if $l\left(H_{\mathrm{m}}^{i}(M)\right)<+\infty$ for $i=0, \cdots$, $d-1$. It is well-known that every generalized Cohen-Macaulay module is an $f$-module and that the converse holds if $A$ is a factor ring of a Cohen-Macaulay ring [3]. But in general, an $f$-module is not a generalized Cohen-Macaulay module. Ferrand and Raynaud [4] have constructed a two-dimensional local integral domain $R$ such that the m-adic completion $\hat{R}$ has a one-dimensional associated prime ideal. Thus, $R$ is an $f$-ring but it is not a generalized Cohen-Macaulay ring.

An s.o.p. $x_{1}, \cdots, x_{d}$ for $M$ is called a standard s.o.p. if $l\left(M / I_{d} M\right)-$ $e\left(I_{d} ; M\right)=l\left(M /\left(x_{1}^{2}, \cdots, x_{d}^{2}\right) M\right)-e\left(x_{1}^{2}, \cdots, x_{d}^{2} ; M\right)$. Trung [11] has shown that $M$ is a generalized Cohen-Macaulay module if and only if there exists a standard s.o.p. for $M$ and that if $x_{1}, \cdots, x_{d}$ is a standard s.o.p., then for all $n_{1}, \cdots, n_{d} \geq 1, l\left(M /\left(x_{1}^{n_{1}}, \cdots, x_{d}^{n_{d}}\right) M\right)-e\left(x_{1}^{n_{1}}, \cdots, x_{d}^{n_{d}} ; M\right)$ is a constant. Therefore $x_{1}, \cdots, x_{d}$ is an unconditioned $p$-s.o.p. for $M$ with respect to the integer $n_{0}=1$. As for the converse, we have the following

Proposition 5. $M$ is a generalized Cohen-Macaulay module if and only if there exists an unconditioned filter regular s.o.p. $x_{1}, \cdots, x_{d}$ such that $l\left(M /\left(x_{1}^{n_{1}}, \cdots, x_{d}^{n_{d}}\right) M\right)$ is a polynomial for all $n_{1}, \cdots, n_{d} \geq 1$. And in this case, $x_{1}, \cdots, x_{d}$ is a standard s.o.p.

Proof. By the above remark it suffices to show the "if" part of the proposition. Let $x_{1}, \cdots, x_{d}$ be an unconditioned filter regular s.o.p. for $M$. Using the notations as in Section 2, by Corollary 4.8 of [1] we have for $n_{1}, \cdots, n_{d} \geq 1$

$$
\left.l\left(M / I_{n(d)} M\right)-e\left(I_{n(d)} ; M\right)=l\left(I_{n(d-1)} M: x_{d}^{n d}\right) / I_{n(d-1)} M\right)
$$

is a polynomial for every permutation of $x_{1}, \cdots, x_{d}$. Then it follows that this difference is independent of $n_{1}, \cdots, n_{d}$. So $x_{1}, \cdots, x_{d}$ is a standard s.o.p. for $M$ and $M$ is a generalized Cohen-Macaulay module.

Remark. The condition that $x_{1}, \cdots, x_{d}$ is a filter regular s.o.p. for every permutation of the sequence $x_{1}, \cdots, x_{d}$ is necessary as the following example shows. Let $A=k \llbracket X, Y, Z \rrbracket /\left(X^{2}, X Y Z, X Z^{2}\right)$ and let $y, z$ be the images of $Y, Z^{2}$ in $A$. Then it is easy to see that $y, z$ is an unconditioned $p$-s.o.p. of $A$ and a filter regular s.o.p. But $A$ is not a generalized CohenMacaulay module since $z, y$ is not a filter regular s.o.p. of $A$. 
Corollary 6. Let $M$ be an f-module. If $M$ is not a generalized CohenMacaulay module then, for every s.o.p. $x_{1}, \cdots, x_{d}$ for $M, l\left(M /\left(x_{1}^{n_{1}}, \cdots, x_{d}^{n_{d}}\right) M\right)$ is never a polynomial for $n_{1}, \cdots, n_{d} \gg 0$.

Proof. Note that there always exists an unconditioned filter regular s.o.p. for $M$ (see [2]). Then the proof is immediate from Proposition 5.

Corollary 7. The following conditions are equivalent:

(i) $M$ is a generalized Cohen-Macaulay module

(ii) Every s.o.p. for $M$ is a p-s.o.p.

(iii) For every s.o.p. $x_{1}, \cdots, x_{d}$ for $M$, $\left.l\left(M / x_{1}^{n_{1}}, \cdots, x_{d}^{n_{d}}\right) M\right)$ is a polynomial for $n_{1}, \cdots, n_{d} \gg 0$.

Proof. Immediate.

§4. The case $\operatorname{dim}(M)=2$

In this section we always assume that $\operatorname{dim}(M)=2$. We will first show that the property of being a p-s.o.p. is stable under permutations.

Lemma 8. Every p-s.o.p. is unconditioned.

Proof. Let $x, y$ be a $p$-s.o.p. for $M$. By Lemma 4 we have

$$
\begin{aligned}
l\left(M /\left(x^{n}, y^{m}\right) M\right) & =l\left(x^{n} M: y^{m} / x^{n} M\right)+m e\left(y ; 0:{ }_{M} x^{n}\right)+n m e(x, y ; M) \\
& =l\left(y^{m} M: x^{n} / y^{m} M\right)+n e\left(x ; 0:{ }_{M} y^{m}\right)+n m e(x, y ; M) .
\end{aligned}
$$

Thus

$$
l\left(y^{m} M: x^{n} / y^{m} M\right)-m e\left(y ; 0:{ }_{M} x^{n}\right)=l\left(x^{n} M: y^{m} / x^{n} M\right)-n e\left(x ; 0:{ }_{M} y^{m}\right),
$$

since $x, y$ is a $p$-s.o.p., it follows that the above difference is a constant, say $k$ for $n, m \gg 0$. Then

$$
\begin{aligned}
& l\left(x^{n} M: y^{m} / x^{n} M\right)=n e\left(x ; 0:{ }_{M} y^{m}\right)+k, \\
& l\left(y^{m} M: x^{n} / y^{m} M\right)=m e\left(y ; 0:{ }_{M} x^{n}\right)+k
\end{aligned}
$$

are polynomials for $n, m \gg 0$ and we get the result by Theorem 1 .

Theorem 9. The following conditions are equivalent:

(i) $x, y$ is a p-s.o.p. for $M$.

(ii) $\left.l\left(M / x^{n}, y^{m}\right) M\right)$ is a polynomial for $n, m \gg 0$.

(iii) $l\left(H_{1}\left(x^{n}, y^{m} ; M\right)\right)$ is a polynomial for $n, m \gg 0$, where $H_{*}(x, y ; M)$ is the homology of the Koszul-Complex $K_{*}(x, y ; A) \otimes{ }_{A} M$ with respect to the elements $x, y$ of $A$. 
Proof. (i) $\Leftrightarrow$ (ii) by Theorem 1 and Lemma 8. (ii) $\Leftrightarrow$ (iii) arises most directly from

$$
l\left(M /\left(x^{n}, y^{m}\right) M\right)-l\left(H_{1}\left(x^{n}, y^{m}, M\right)\right)=n m e(x, y ; M)-l\left(0:_{M}\left(x^{n}, y^{m}\right)\right)
$$

which is a polynomial for $n, m \gg 0$.

Let $N \subseteq M$ a submodule and $J \subseteq A$ an ideal. We set

$N:\langle J\rangle=\left\{a \in M ; a J^{k} \subseteq N\right.$ for some $\left.k \geq 1\right\}$.

We will see that for a filter regular s.o.p., the property of being a p-s.o.p. can be expressed in terms of only one element,

Proposition 10. Let $x, y$ be a filter regular s.o.p. for $M$. Then the following conditions are equivalent:

(i) $l\left(M /\left(x^{n}, y^{m}\right) M\right)$ is a polynomial for $n, m \gg 0$.

(ii) There exists a positive integer $k$ such that

$$
y^{m}\left(y^{k} M:\langle\mathfrak{m}\rangle\right)+0:\langle\mathfrak{m}\rangle=y^{k+\mathfrak{m}} M:\langle\mathfrak{m}\rangle
$$

for all $m \geq 0$.

Proof. (i) $\Rightarrow$ (ii). Note that if $x, y$ is a filter regular s.o.p. then $x^{n}, y^{m}$ is also a filter regular s.o.p. for all $n, m \geq 1$. By Lemma 4 and Corollary 4.8 of [1] we get

$$
\begin{aligned}
l\left(M /\left(x^{n}, y^{m}\right) M\right)-n m e(x, y ; M) & =l\left(x^{n} M: y^{m} / x^{n} M\right) \\
& =l\left(y^{m} M: x^{n} / y^{m} M\right)+n e\left(x ; 0:{ }_{M} y^{m}\right) .
\end{aligned}
$$

This shows that the above difference is a polynomial depending only on $n$. Thus there exists a positive integer $k$ such that $l\left(y^{m} M: x^{n} / y^{m} M\right)$ is a constant for all $n, m \geq k$. As $x, y$ is a $p$-s.o.p. we can choose a sufficiently large $k$ such that $y^{m} M: x^{n}=y^{m} M: \mathfrak{m}^{k}=y^{m} M:\langle\mathfrak{m}\rangle$ and $0:\langle y\rangle=0:{ }_{M} y^{k}$ for $n, m \geq k$. We have

$$
\begin{aligned}
l\left(y^{m} M: x^{n} / y^{m} M\right) & =l\left(y^{\mathfrak{m}} M:\langle\mathfrak{m}\rangle \mid y^{m} M\right) \\
& =l\left(y^{m} M:\langle\mathfrak{m}\rangle \mid y^{m} M+0:\langle\mathfrak{m}\rangle\right)+l\left(y^{m} M+0:\langle\mathfrak{m}\rangle \mid y^{m} M\right) \\
& =l\left(y^{m} M:\langle\mathfrak{m}\rangle \mid y^{m} M+0:\langle\mathfrak{m}\rangle\right)+l(0:\langle\mathfrak{m}\rangle),
\end{aligned}
$$

since, by Lemma 2 (ii)

$$
y^{m} M+0:\langle\mathfrak{m}\rangle\left|y^{m} M \simeq 0:\langle\mathfrak{m}\rangle\right| y^{m} M \cap 0:\langle\mathfrak{m}\rangle \simeq 0:\langle\mathfrak{m}\rangle .
$$

Thus $l\left(y^{m} M:\langle\mathfrak{m}\rangle / y^{m} M+0:\langle\mathfrak{m}\rangle\right)$ is a constant for $m \geq k$. Now we consider the mapping 


$$
f_{m}: y^{k} M:\langle\mathfrak{m}\rangle\left|y^{k} M+0:\langle\mathfrak{m}\rangle \longrightarrow y^{k+m} M:\langle\mathfrak{m}\rangle\right| y^{k+m} M+0:\langle\mathfrak{m}\rangle
$$

defined by $f_{m}(a)=a \cdot y^{m}$ for $a \in M$. We will show now that $f_{m}$ is injective for all $m \geq 0$. In fact, since

$$
\operatorname{ker}\left(f_{m}\right)=\left(\left(y^{k} M:\langle m\rangle\right) \cap\left(y^{k} M+0:{ }_{M} y^{m}\right)\right) / y^{k} M+0:\langle\mathfrak{m}\rangle
$$

and

$$
\left(y^{k} M:\langle m\rangle\right) \cap\left(y^{n} M+0:{ }_{M} y^{m}\right)=y^{k} M+\left(y^{k} M:\langle m\rangle\right) \cap\left(0:{ }_{M} y^{m}\right),
$$

we only need to show that $\left(0:{ }_{M} y^{m}\right) \cap\left(y^{k} M:\langle m\rangle\right) \subseteq 0:\langle\mathfrak{m}\rangle$. Let $a \in\left(0:_{M} y^{m}\right)$ $\cap\left(y^{k} M:\langle\mathfrak{m}\rangle\right)$, for arbitrary $b \in \mathfrak{m}^{k}, a b=y^{k} c$ for some $c \in M$. As $a y^{k}=0$, $0=a y^{k} b=y^{2 k} c$. Thus $c \in 0:{ }_{M} y^{2 k}=0:{ }_{M} y^{k}=0:\langle y\rangle$ and $a b=y^{k} c=0$ for all $b \in \mathfrak{m}^{k}$. So it follows that $a \in 0:{ }_{M} \mathfrak{m}^{k}=0:\langle\mathfrak{m}\rangle$. Since $l\left(y^{k+m} M:\langle\mathrm{m}\rangle\right.$ $\left.\mid y^{k+m} M+0:\langle\mathfrak{m}\rangle\right)$ is a constant and $f_{m}$ is injective for $m \geq 0$, it follows that $f_{m}$ is surjective for all $m \geq 0$ and this proves that $y^{m}\left(y^{k} M:\langle n I\rangle\right)+$ $0:\langle m\rangle=y^{k+m} M:\langle m\rangle$ for all $m \geq 0$.

(ii) $\Rightarrow$ (i). By Theorem 1 and Lemma 8 it is enough to show that $y, x$ is a $p$-s.o.p. There exists integers $t, s$ such that $y^{k} M:\langle m\rangle=y^{k} M: x^{t}$ and $0:\langle x\rangle=0:{ }_{x} x^{s}$. Let $n_{0}=\max \{k, t, s\}$. Then, for all $m \geq n_{0}$,

$$
\begin{aligned}
y^{m} M:\langle\mathfrak{m}\rangle & =y^{m-k}\left(y^{k} M:\langle\mathfrak{m}\rangle\right)+0:\langle\mathfrak{M l}\rangle \\
& =y^{m-k}\left(y^{k} M: x^{n_{0}}\right)+0:\langle\mathfrak{l}\rangle \subseteq y^{m} M: x^{n_{0}} .
\end{aligned}
$$

This completes the proof of Proposition 10.

The Proposition 10 has the following consequences.

Corollary 11. Let $x, y$ be a filter regular s.o.p. for $M$. If $l\left(M /\left(x^{n}, y^{n}\right) M\right)$ is a polynomial for $n, m \gg 0$ then, for every $z \in A$ such that $z, y$ is a s.o.p. for $M, z, y$ is a filter regular s.o.p. for $M$ and $l\left(M /\left(z^{n}, y^{m}\right) M\right)$ is a polynomial for $n, m \gg 0$.

Proof. By Proposition 10 we need only to show that if $z, y$ is a s.o.p. for $M$, then $z, y$ is a filter regular s.o.p. for $M$. In fact, we have

$$
0:\langle\boldsymbol{z}\rangle \subseteq y^{m+k} M:\langle z\rangle=y^{m+k} M:\langle\mathfrak{m}\rangle=y^{m}\left(y^{k} M:\langle\mathfrak{m}\rangle\right)+0:\langle\mathfrak{m}\rangle
$$

for all $m \geq 0$ and $k$ as in Proposition 10. It follows that

$$
0:\langle z\rangle \subseteq \bigcap_{m=0}^{\infty}\left(y^{m}\left(y^{k} M:\langle\mathfrak{m}\rangle\right)+0:\langle\mathfrak{m}\rangle\right)=0:\langle\mathfrak{m}\rangle
$$

by Krull's Intersection Theorem. Hence we can conclude that $0:\langle z\rangle=$ G: $\langle\mathfrak{m}\rangle$. 
As for the next corollary, we recall a notation from [6]. Let $x, y$ be an s.o.p. of $A$ and $t$ an indeterminate over $A$. Then one call the graded algebra $R_{\mathrm{m}}(x)=\bigoplus_{n=-\infty}^{+\infty}\left(x^{n} A:\langle\mathfrak{m}\rangle\right) t^{n}$ the $\mathrm{m}$-relative Rees ring with respect to the ideal $x A$. Let $R(x)=\bigoplus_{n=-\infty}^{+\infty}\left(x^{n} A\right) t^{n}$ be the ordinary Rees ring of $A$ with respect to $x A$.

Corollary 12. Let $M=A$ and $x, y$ form an s.o.p. of $A$. Then the following conditions are equivalent:

(i) $R_{\mathrm{n}}(x)$ is finitely generated over $R(x)$.

(ii) $\operatorname{depth}(A)>0$ and $l\left(A /\left(x^{n}, y\right) A\right)$ is a polynomial for $n, m \gg 0$.

Proof. It is well-known [6] that (i) is equivalent to the following condition

(i') There exists a positive integer $k$ such that for all $n \geq 0 x^{n}\left(x^{k} A:\langle\mathfrak{m}\rangle\right)$ $=x^{n+k} A:\langle\mathfrak{m}\rangle$.

Thus, (ii) $\Rightarrow\left(i^{\prime}\right)$ by Proposition 10 . (i) $\Rightarrow$ (ii) follows from the Proposition 10 and

$$
0:{ }_{\mu} y \subseteq \bigcap_{n=0}^{\infty}\left(x^{n+k} A:\langle y\rangle\right)=\bigcap_{n=0}^{\infty}\left(x^{n+k} A:\langle\mathrm{m} \mathrm{t}\rangle\right)=\bigcap_{n=0}^{\infty}\left(x^{n}\left(x^{k} A:\langle\mathrm{m}\rangle\right)\right)=0 .
$$

Acknowlegement. This note was written while the author was visiting the Department of Mathematics of the University of Genova by a grant of Consiglio Nazionale delle Ricerche. He would like to thank both institutions for support and hospitality. Also, he would like to thank G. Valla and N.V. Trung for their useful suggestions.

\section{REFERENCES}

[1] Auslander, M. and D. A. Buchsbaum, Codimension and multiplicity, Ann. Math., 68 (1958), 625-657.

[2] Brodmann, M., Kohomologische Eigenschaften von Aufblasungen an lokalen vollständigen Durchschnitten, Habilitationsschrift, Münster 1980.

[ 3 ] Cuong, N. T., Trung, N. V. and P. Schenzel, Verallgemeinerte Cohen-Macaulay Moduln, Math. Nachr., 85 (1978), 57-73.

[4] Ferrand, D. and M. Raynaud, Fibres formelles d'un anneau local Noetherien, Ann. Sc. Ecole Norm. Sup., 3 (1970), 295-311.

[5] Garcia Roig, J-L and D. Kirby, On the Koszul homology modules for the powers of a multiplicity system, Mathematika, 33 (1986), 96-101.

[6] Schenzel, P., Finiteness of relative Rees ring and Asymptotic Prime Divisor, Math. Nachr., 129 (1986), 123-148.

[ 7 ] Serre, J-P., Algèbre locale: Multiplicités, Lecture Notes in Math. No. 11, 1965.

[ 8 ] Stückrad, J. and W. Vogel, Eine Verallgemeinerung der Cohen-Macaulay Ringe und Anwendungen auf ein Problem der Multiplizitätstheorie, J. Math. Kyoto Univ., 13 (1973), 513-528. 
[9] Sharp, R. Y. and M. A. Hamieh, Lengths of certain generalized fraction, J. pure Appl. Algebra, 38 (1985), 323-336.

[10] Trung, N. V., Absolutely superficial sequence, Math. Proc. Cambrige Phil. Soc., 93 (1983), 35-47.

[11] - Toward a theory of generalized Cohen Macaulay modules, Nagoya Math. J., 102 (1986), 1-49.

[12] Cuong, N. T., Generalized Cohen-Macaulay modules with non-Cohen-Macaulay locus of positive dimension, preprint.

[13] Huneke, C., Theory of $d$-sequences and powers of ideals, Adv. in Math., 46 (1982), 249-279.

Institute of Mathematics

P.O. Box 631, Bo Ho

Hanoi

Vietnam 